\title{
Explore postgraduate biomedical engineering course integration between medical signal processing and drug development: example for drug development in brain disease
}

\author{
Yin Tian*, Li Yang, Zhongyan Wang, Huiling Zhang, Wei Xu, Shuxing Zheng, Haying \\ Zhang, Dechun Zhao \\ Bio-information College, ChongQing University of Posts and Telecommunications, ChongQing 400065, China \\ *Corresponding Author: E-mail: tiany20032003@163.com; Tel.: +86-23-62460536; Fax: +86-23-62460536 \\ Received: June 28, 2016; Published: July 03, 2016
}

\begin{abstract}
Medical signal processing is a compulsory course in our university's undergraduate biomedical engineering programme. Recently, application of medical signal processing in supporting new drug development has emerged as a promising strategy in neurosciences. Here, we discuss the curriculum reformation in biomedical signal processing course in the context of drug development and application in central nervous system, with a particular emphasis in knowledge integration.
\end{abstract}

\section{Keywords}

Biomedical signal processing; drug development; course integration; curriculum reformation

\section{Introduction}

Modern medicine development technology is a multi-disciplinary topic and has received considerable attention in recent years. In order to develop a solid foundation, wide knowledge, high cultural quality, and strong adapt ability of multi-disciplinary talent, we are carrying education reform on personnel training mode and optimized programme curriculum in the major of biomedical engineering. The new curriculum aims to enhance strong links between theories and practical applications with wide range of content covering multi-disciplinary topic areas. Without a carefully planned curriculum, it is difficult to cover this course with the allocated contact hours. Therefore, this article will explore the theoretical and experimental aspects of combining biomedical signal processing and central nervous system (CNS) drug development [1].

\section{Curriculum integration}

The drug development technology using medical signal processing is an international frontier in the field of modern medical technology. We propose to focus on the application of medical signal processing on pharmacokinetics and pharmacodynamics of the drug development in teaching, highlighting combination with the implementation principles, the technical methods and applications. Pharmacokinetic (PK) is 
mainly a quantitative study on drug processing in organisms (including absorption, distribution, metabolism and excretion) and described the dynamic regularity of drug in the body by the mathematical principle and methods. Pharmacodynamics (PD) examines the effect, mechanism and quantity discipline of the drug on the body, namely under the action of drugs, the body organs physiological function and metabolic activity of cells. Both PK and PD make up the foundation of modern pharmacology study. Combination of these disciplines could indicate the time process of pharmacological effects caused by a given certain dosage. Study of PK/PD relationship not only helps to guide the clinical medication correctly, but also explore mechanisms of drug action, new drug evaluation and the development of new preparations [2].

Recently, a variety of integrated application of brain imaging technology has become a development trend of modern drug research and development, especially in the cranial nerve disease (such as Alzheimer's and Parkinson's disease) and mental illness (such as schizophrenia/depression) in drug development for biomarkers. Electroencephalogram (EEG) and magnetoencephalogram (MEG) can provide high temporal resolution of cognitive activity information. Functional magnetic resonance imaging (fMRI) and positron emission layer tracing technique (PET) can provide high spatial resolution information. Mapping brain structure by registration and fusion of these two kinds of information (EEG/MEG and fMRI /PET) has become an important technical route.

This course may be considered to offer at the postgraduate level. The students have already learned in their undergraduate courses in physiological basis, signal and system, biomedical signal processing, PET imaging technology, as well as biomedical electronics and detection technology. The study of the theory of these courses has laid a solid foundation. The setting of the proposed course contents are as follows: (1) brain disease of physiology and disease, (2) drug development basis on PK and PD, (3) EEG signal acquisition and analysis and (4) combination of EEG and CNS drug development.

\section{Combining with teaching and experiment}

From the curriculum setting, this course focuses on the biomedical signal processing as a means of technical analysis. During the evaluation of drug performance in the course of central nervous system (CNS) drug development, EEG signals and metabolic signals elicited by brain activity are recorded and analysed in the time domain and frequency domain using the time-frequency analysis method associated with cognitive activities to brain connectomes. These involve strong theoretical knowledge and practical techniques in biomedical signal processing and medical image processing. To improve students' initiative to acquire knowledge and problem solving ability, it is important to development a holistic teaching method. To this end, we combine both the teaching in theory and experiments as well as substantiate further with the training on preliminary scientific research ability and practical applications of the medical instrument.

\section{Existing problems and solving suggestion}

The difficulties in the integration of CNS drug development and biomedical signal processing are: wide course content span involving multidisciplinary sciences (e.g., signal and system, signal processing and physiology, etc.), too close to the current most advanced science and technology and insufficient contact hours. Many students might have already forgotten the basic knowledge they have learned in prerequisite courses taken in the previous semesters. It may be difficult for them to grasp the new contents in the first instance. Therefore, the teacher should offer revision lessons at the beginning of the course, so that the students can easily get up to speed in their learning. Interactive teaching is highly recommended. Teacher can then adjust the speed of the lessons to ensure effectiveness of the teaching with sufficient explanation 
in the course materials in order to effectively help students to digest and consolidate what they learned. At the same time, through the hands on experiment data processing, the student's ability to analyse actual data could be improved, and conducive to the follow-up student's self-experimental design. In addition to explain the new knowledge points in the teaching, the teacher are encouraged to be proactive to help students to solve practical PK/PD combined with signal processing analysis. The use of existing programming software (MATLAB), open software EMSE, EEGLAB analysis software to carry out the signal processing analysis is highly recommended and should be accomplished in an interactive manner. This helps not only reducing the analysis time, but also minimizing the needs to perform tedious numerical operations. At the same time, the students could save time in the interpretation of the analysis results.

\section{Conclusion}

Multidisciplinary curriculum integration is the current focus of the teaching reform in colleges and universities. Using a postgraduate biomedical engineering course as an example, we have explored the educational reform by the integration of both biomedical signal processing and PD/PD on CNS drug development.

\section{Acknowledgements}

We thank the support from CQUPT's educational projects (\#YKC2015007 and \#XJG1519).

\section{References}

[1] Y. Tian, Z.D. Ding, Z.Y. Wang, H.L. Zhang, $4^{\text {th }}$ World Conference on Physico-Chemical Methods in Drug Discovery and Development (September, 2015).

[2] L.H. Reddy, D. Bazile, Advanced Drug Delivery Reviews 71 (2013) 34-57.

C 2016 by the authors; licensee IAPC, Zagreb, Croatia. This article is an open-access article distributed under the terms and conditions of the Creative Commons Attribution license (http://creativecommons.org/licenses/by/3.0/) (cc)) EY 